\title{
Infiltração intraarticular de plasma rico em plaquetas versus ácido hialurônico em pacientes com osteoartrose primária do joelho: Ensaio clínico randomizado com resultados preliminares*
}

\section{Intraarticular Infiltration of Platelet-Rich Plasma versus Hyaluronic Acid in Patients with Primary Knee Osteoarthritis: Preliminary Results from a Randomized Clinical Trial}

\author{
Gustavo Gonçalves Arliani ${ }^{10}$ Thomas Stravinskas Durigon ${ }^{1}$ (1) João Paulo Pedroso ${ }^{1}$ (1) \\ Gabriel Ferraz Ferreira ${ }^{10}$ Daniel Oksman ${ }^{10}$ Victor Otávio Oliveira1일 \\ ${ }^{1}$ Departamento de Ortopedia e Traumatologia, Instituto Prevent
Senior, São Paulo, SP, Brasil \\ Endereço para correspondência Gabriel Ferraz Ferreira, MD, MSc, Rua \\ Cerro Corá, 585, Sala 605, Torre 1, São Paulo, SP, Brasil \\ (e-mail: gabriel.ferraz38@yahoo.com.br).
}

Rev Bras Ortop 2022;57(3):402-408.

\section{Resumo \\ Objetivo Comparar o efeito da infiltração intraarticular do plasma rico em plaqueta com a do ácido hialurônico no tratamento de pacientes com osteoartrose primária de joelho. \\ Métodos Realizou-se um ensaio clínico randomizado com 29 pacientes, sendo um grupo submetido à infiltração com ácido hialurônico (controle) e o outro com plasma rico em plaquetas. Os desfechos clínicos avaliados foram a escala visual analógica da dor; o questionário Western Ontario and McMaster Universities Arthritis Index (WOMAC), antes e depois da intervenção; e os efeitos adversos após as aplicações. Utilizou-se os testes do qui-quadrado e exato de Fisher para as variáveis categóricas, e o teste t de Student, análise de variância, e Wilcoxon para as variáveis contínuas, através do software R. \\ Resultados A análise independente de cada grupo revelou uma diferença estatística nos meses iniciais, com melhora dos escores de dor e função; porém, com piora no $6^{\circ}$ \\ Palavras-chave \\ - osteoartrite do joelho \\ - ácido hialurônico \\ - infiltração mês após o procedimento. Não houve diferença dos desfechos avaliados entre os grupos que foram submetidos à infiltração com ácido hialurônico ou com plasma rico em plaquetas. Não houve efeito adverso grave ou reação alérgica durante todo o seguimento.}

Trabalho desenvolvido no Departamento de Ortopedia e Traumatologia, Instituto Prevent Senior, São Paulo, Brasil.

recebido

05 de Junho de 2020

aceito

17 de Setembro de 2020

Publicado on-line

Abril 19, 2021
DOI https://doi.org/ 10.1055/s-0041-1724082. ISSN 0102-3616.
(C) 2021. Sociedade Brasileira de Ortopedia e Traumatologia. All rights reserved.

This is an open access article published by Thieme under the terms of the Creative Commons Attribution-NonDerivative-NonCommercial-License, permitting copying and reproduction so long as the original work is given appropriate credit. Contents may not be used for commercial purposes, or adapted, remixed, transformed or built upon. (https://creativecommons.org/ licenses/by-nc-nd/4.0/)

Thieme Revinter Publicações Ltda., Rua do Matoso 170, Rio de Janeiro, RJ, CEP 20270-135, Brazil 


\section{Abstract}

\section{Keywords}

- osteoarthritis, knee

- hyaluronic acid

- infiltration
Conclusão A infiltração intraarticular com ácido hialurônico ou plasma rico em plaquetas nos joelhos dos pacientes com gonartrose primária apresentou melhora temporária dos sintomas de função e dor. Não houve diferença entre as duas intervenções.

Objective The present study aimed to compare the effects of intraarticular infiltration of platelet-rich plasma with those of hyaluronic acid infiltration in the treatment of patients with primary knee osteoarthritis.

Methods A randomized clinical trial was conducted with 29 patients who received an intraarticular infiltration with hyaluronic acid (control group) or platelet-rich plasma. Clinical outcomes were assessed using the visual analog scale for pain and the Western Ontario and McMaster Universities Arthritis Index (WOMAC) questionnaire before and after the intervention. In addition, the posttreatment adverse effects were recorded. Categorical variables were analyzed using the chi-square and Fisher exact tests, whereas continuous variables were analyzed using the Student $t$ test, analysis of variance, and the Wilcoxon test; all calculations were performed with the Stats package of the R software.

Results An independent analysis of each group revealed a statistical difference within the first months, with improvement in the pain and function scores, but worsening on the $6^{\text {th }}$ month after the procedure. There was no difference in the outcomes between the groups receiving hyaluronic acid or platelet-rich plasma. There was no serious adverse effect or allergic reaction during the entire follow-up period.

Conclusion Intraarticular infiltration with hyaluronic acid or platelet-rich plasma in patients with primary knee gonarthrosis resulted in temporary improvement of functional symptoms and pain. There was no difference between interventions.

\section{Introdução}

A osteoartrose $(\mathrm{OA})$ do joelho é uma doença de caráter degenerativo, predominantemente no sexo feminino, que provoca a destruição da cartilagem articular de forma progressiva. Esta doença leva à deformidade da articulação, potencialmente com desequilíbrio muscular, ligamentar, e alterações principalmente nas regiões de maior carga, com sinais radiográficos típicos, tais como esclerose óssea, cistos e osteófitos. ${ }^{1-3}$

A articulação do joelho com $\mathrm{OA}$ apresenta grande impacto no desempenho físico e é considerada uma das 10 principais causas de incapacidade no mundo. Os tratamentos conservadores padrão para osteoartrose do joelho incluem: perda de peso, exercícios físicos, uso de antiinflamatórios não esteroides, analgésicos, injeção intraarticular de ácido hialurônico (AH) e glicocorticoides. ${ }^{4}$

O AH é uma das opções utilizadas no tratamento da doença articular degenerativa. Trata-se de um glicosaminoglicano que atua na matriz extracelular proporcionando maior lubrificação e proteção das articulações.

No entanto, recentemente, as injeções ortobiológicas surgiram como uma opção potencialmente segura e eficaz no tratamento da osteoartrose do joelho; entre elas, podemos citar o concentrado de medula ósseo (CMO), as células tronco mesenquimais (CTM) e o plasma rico em plaquetas (PRP).
O PRP é composto por plasma com uma concentração de plaquetas acima do valor normal. ${ }^{5}$ Dependendo do método utilizado no processamento do PRP, este também pode conter células brancas do sangue em concentrações acima do valor normal. ${ }^{6}$ Plaquetas e glóbulos brancos são fontes de elevadas concentrações de citocinas, que apresentam papel bem documentado no controle de uma série de processos relacionados com a regeneração tecidual. Esses processos incluem a movimentação e proliferação celular, angiogênese, controle da inflamação e síntese de colágeno. ${ }^{6}$

As plaquetas, além de realizar a hemostasia local, contêm uma abundância de fatores de crescimento e citocinas, que são cruciais na cicatrização de tecidos moles e mineralização óssea. ${ }^{7}$ Além disso, estas liberam uma série de proteínas responsáveis pela atração de macrófagos, células-tronco mesenquimais e osteoblastos, que não só promovem a remoção de tecidos necróticos, mas também aceleram a regeneração tecidual. ${ }^{4}$

Recentemente, alguns estudos foram realizados investigando os possíveis efeitos benéficos do PRP no tratamento de doenças crônicas, incluindo epicondilite lateral e fascite plantar. ${ }^{4}$ No entanto, a maioria dos estudos presentes na literatura com uso de PRP são não randomizados e com amostra insuficiente.

O objetivo do presente estudo é determinar o efeito do tratamento com aplicação intraarticular de PRP nos pacientes 
com diagnóstico de OA e compará-lo ao efeito do tratamento com AH em relação aos desfechos de dor e função.

\section{Método}

O presente estudo é um ensaio clínico randomizado com 29 pacientes incluídos consecutivamente. Todos os pacientes que participaram deste estudo concordaram e assinaram o termo de consentimento livre e esclarecido.

O estudo foi conduzido de acordo com a Declaração de Helsinque e as Diretrizes de Boas Práticas Clínicas. O protocolo foi aprovado pelo comitê de ética local (Parecer da Plataforma Brasil: 3.293.253)

\section{Seleção dos pacientes}

A amostra total contou com a presença de 29 pacientes de ambos os gêneros, com idade entre 49 e 75 anos, que preencheram os critérios diagnósticos clínicos e radiográficos da American College of Reumathology (ACR) para a OA do joelho e classificados como graus II e III de Kellgren-Lawrence. ${ }^{2}$

Os critérios de exclusão foram: cirurgias prévias no joelho acometido a qualquer tempo ou cirurgia ortopédica nos membros inferiores nos últimos 12 meses; infiltração prévia de $\mathrm{AH}$ ou corticoide nos últimos 3 meses; casos avançados de OA (graus IV e V); diagnóstico de doenças autoimunes ou reumatológicas; índice de massa corpórea (IMC) maior ou igual a 35; OA secundária (ex: fraturas, neoplasias); história de doenças transmissíveis agudas ou crônicas; diabetes tipo I ou II de difícil controle ou insulino-dependentes; diagnóstico de coxartrose, mediante a alteração de exame físico ou imagem radiográfica; infecção ativa ou história de infecção na articulação a ser infiltrada; desvio axial em varo de 10 graus, valgo de 15 graus, ou discrepância de membros inferiores de $1 \mathrm{~cm}$; uso de anticoagulantes ou imunossupressores; descontinuidade no uso de condroprotetores orais nos últimos 3 meses e função renal e/ou hepática com valores fora da normalidade.

Todos os pacientes incluídos no estudo tinham diagnóstico confirmado de $\mathrm{OA}$ do joelho e foram submetidos ao tratamento conservador com fisioterapia, alongamentos e uso de analgésicos pelo período mínimo de 6 meses antes do início do estudo. A avaliação da $\mathrm{OA}$ foi realizada com imagens radiográficas dos joelhos em duas projeções com carga: anteroposterior e perfil.

Os exames solicitados nas visitas de pré-seleção foram: exames bioquímicos (transaminase glutâmico-oxalacética [TGO], transaminase glutâmico-pirúvica [TGP], gama-glutamil transferase [GGT], glicose de jejum, creatinina, sódio, potássio, hemoglobina glicada, e hemograma completo), sorologia para doenças transmissíveis, ressonância magnética do joelho acometido, radiografia bilateral de joelho e radiografia panorâmica de membros inferiores.

\section{Randomização}

A randomização dos pacientes foi feita por meio do sistema Research Randomizer. ${ }^{8}$ Desse modo, o estudo contou com dois braços: grupo estudo, no qual foi realizada a aplicação intraarticular de PRP, e o grupo controle, com aplicação do AH.

\section{Método de aplicação}

Os pacientes de ambos os braços do estudo foram agendados ambulatorialmente na semana seguinte para aplicação da medicação. Os pacientes do grupo controle foram submetidos a uma aplicação única de Synvisc One Hylan G-F20 (Lancaster, Pennsylvania, Estados Unidos) intraarticular no joelho, seguindo medidas específicas de assepsia e antissepsia.

Os pacientes do grupo estudo, na chegada ao hospital, foram direcionados para o setor de coleta de sangue, onde foi coletado uma amostra de $15 \mathrm{ml}$ de sangue por acesso periférico de modo estéril em tubo específico, sendo então transportada em temperatura controlada para processamento em laboratório localizado no mesmo hospital.

A coleta foi centrifugada a 1.500 rotações por minuto pelo período de 5 minutos em temperatura ambiente. A partir daí, a amostra foi quantificada e considerada aceitável se apresentasse o dobro do basal da amostra inicial.

Após a obtenção do PRP na quantidade de aproximadamente $5 \mathrm{ml}$, este foi direcionado para o procedimento de infiltração no joelho do paciente, que foi realizado na sala de pequenos procedimentos cirúrgicos. O PRP foi aplicado através de uma punção intraarticular do joelho. Todo o processo foi realizado através do sistema Autologous Conditioned Plasma (ACP) da empresa Arthrex (Arthrex Inc., Naples, FL, EUA). 0 processo de aplicação foi repetido nas duas semanas seguintes, 7 e 14 dias após a primeira infiltração, respectivamente, somando um total de três infiltrações de PRP.

\section{Seguimento clínico e avaliação dos desfechos}

Os investigadores coletaram dados dos participantes, tais como: idade, lateralidade, índice de massa corpórea (IMC), edema e rigidez no joelho submetido à intervenção. Os pacientes do grupo estudo seguiram a mesma frequência de acompanhamento do grupo controle após a terceira aplicação, e ambos os grupos foram acompanhados pelo período de 6 meses.

O seguimento padronizado contou com monitoramento através de visitas ambulatoriais médicas totalizando 5 visitas no período de 6 meses: a primeira em 1 semana e as seguintes em 2 semanas, 1 mês, 3, e 6 meses, e outros 2 contatos telefônicos nos meses 2 e 4 após a aplicação.

Além disso, aplicou-se o escore de Western Ontario and McMaster Universities Arthritis Index (WOMAC) nos seguintes tempos: pré-infiltração, 1, 3, e 6 meses após a infiltração. A aplicação da escala visual analógica (EVA) da dor foi realizada nos intervalos de 2 e 4 meses após a aplicação.

\section{Análise estatística}

A análise estatística foi feita pelo com o do software de estatística R ( $R$ Foundation for Statistical Computing, Viena, Áustria). ${ }^{9}$ As variáveis contínuas foram mensuradas através da análise descritiva pela média e desvio padrão, e testadas quanto à sua distribuição seguindo a curva normal ou não, pelo teste de Shapiro. ${ }^{10}$ As variáveis categóricas foram apresentadas de acordo com a sua proporção.

Na comparação intragrupo, utilizaram-se os testes análise de variância(ANOVA) com diferença mínima significativa de Fisher (least significant difference $)^{11}$ para avaliar se houve diferença no 
Tabela 1 Características dos pacientes incluídos no estudo

\begin{tabular}{|l|l|l|l|}
\hline & $\begin{array}{l}\text { Plama rico } \\
\text { em plaquetas }\end{array}$ & $\begin{array}{l}\text { Ácido } \\
\text { hialurônico }\end{array}$ & $\begin{array}{l}\text { Valor } \\
\text { de } p\end{array}$ \\
\hline $\begin{array}{l}\text { Número de } \\
\text { pacientes }\end{array}$ & 14 & 15 & - \\
\hline IMC & $28.3\left(2.9^{*}\right)$ & $28.1\left(3.9^{*}\right)$ & 0.60 \\
\hline $\begin{array}{l}\text { Idade (anos), } \\
\text { média (DP) }\end{array}$ & $62.78\left(6.10^{*}\right)$ & $63.40\left(4.99^{*}\right)$ & 0.77 \\
\hline Sexo & $\begin{array}{l}11 \text { mulheres } \\
3 \text { homens }\end{array}$ & $\begin{array}{l}13 \text { mulheres } \\
2 \text { homens }\end{array}$ & 0.93 \\
\hline $\begin{array}{l}\text { Lateralidade } \\
\text { Direito }=7\end{array}$ & $\begin{array}{l}\text { Direito }=11 \\
\text { Esquerdo }=4\end{array}$ & 0.36 \\
\hline $\begin{array}{l}\text { Classificação } \\
\text { radiográfica }\end{array}$ & $\begin{array}{l}\text { Grau II }=9 \\
\text { Grau III }=5\end{array}$ & $\begin{array}{l}\text { Grau II }=9 \\
\text { Grau III }=6\end{array}$ & 1 \\
\hline $\begin{array}{l}\text { Edema } \\
\text { no joelho }\end{array}$ & $\begin{array}{l}\text { Sim }=1 \\
\text { Não }=13\end{array}$ & $\begin{array}{l}\text { Sim }=2 \\
\text { Não }=13\end{array}$ & 1 \\
\hline $\begin{array}{l}\text { Rigidez } \\
\text { no joelho }\end{array}$ & $\begin{array}{l}\text { Sim }=1 \\
\text { Não }=13\end{array}$ & $\begin{array}{l}\text { Sim }=2 \\
\text { Não }=13\end{array}$ & 1 \\
\hline
\end{tabular}

Abreviatura: DP, desvio-padrão; IMC, índice de massa corpórea. Fonte: Prevent Senior São Paulo.

escore WOMAC, e o teste t de Student pareado na análise da EVA da dor.

Já na mensuração da diferença intergrupos, realizaram-se os testes t de Student ${ }^{12}$ para as variáveis paramétricas, e o teste de Mann-Whitney ${ }^{13}$ para as não paramétricas. A avaliação das variáveis categóricas entre o grupo estudo e o grupo controle foi realizada através do teste do Qui quadrado ${ }^{14}$ ou pelo teste exato de Fisher. ${ }^{15}$

\section{Resultados}

Não houve nenhuma perda de pacientes durante o seguimento. Os grupos de controle e de estudo foram homogêneos, sem diferença estatística entre os parâmetros mensurados, conforme demonstrado na - Tabela 1.

A comparação entre os grupos estudo e controle em relação ao desfecho de função (WOMAC) não apresentou diferenças estatísticas entre eles, desde o grau pré-intervenção até com 6 meses de acompanhamento (-Tabela 2).

O escore de dor avaliado pela EVA também não apresentou diferença estatística no $2^{\circ}(p=0.50)$ e $4^{\circ}(p=0.45)$ mês após a aplicação intraarticular, como demonstrado na - Figura 1.

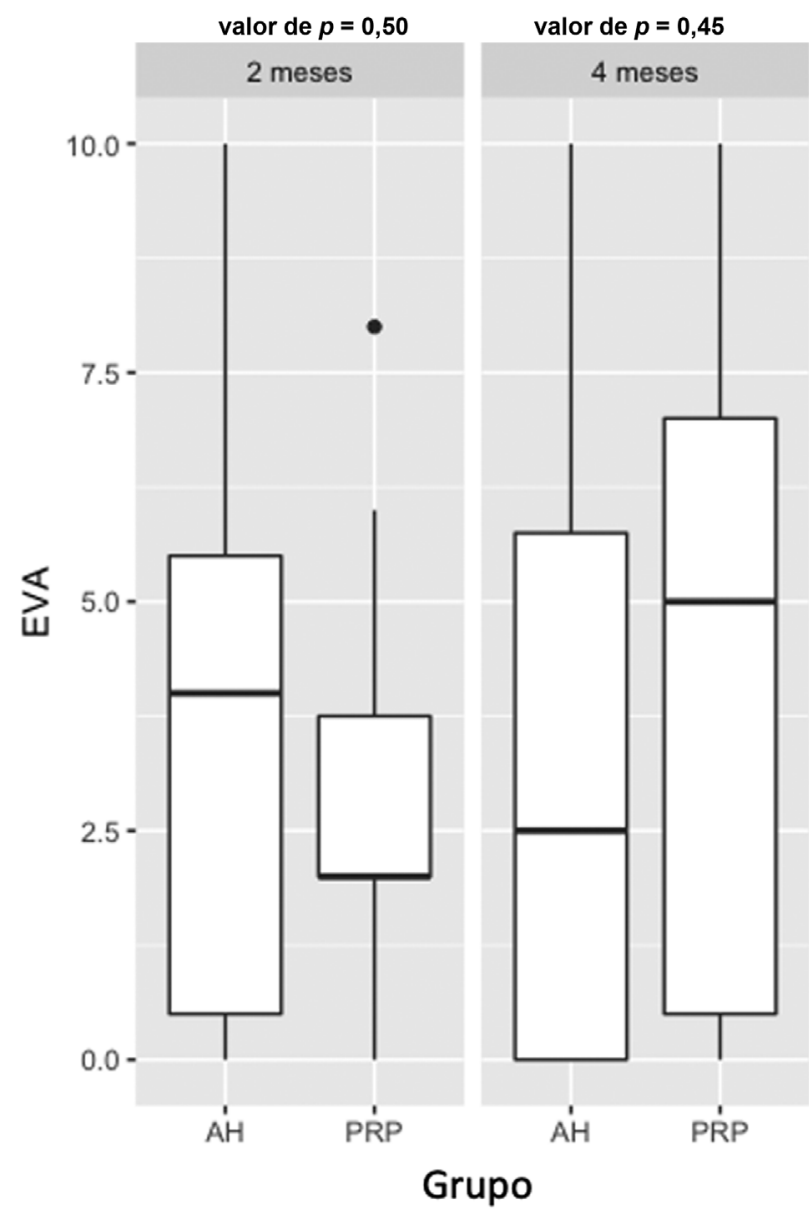

Fig. 1 Distribuição da escala visual analógica da dor entre os grupos ácido hialurônico e plasma rico em plaquetas.

Na avaliação intragrupos, observou-se uma melhora na função após o procedimento, porém com piora no último mês de avaliação. No grupo que realizou a infiltração com PRP, houve diferença estatística entre o WOMAC inicial e 3 meses após o procedimento $(p<0.05)$. Também se observou uma diferença entre o $1^{\circ}$ e $6^{\circ}$ mês do procedimento, evidenciando um aumento no escore $(p<0.05)$. Outra diferença encontrada foi no critério de dor, com uma diferença média da EVA de 1.64 entre o $2^{\circ}$ e o $4^{\circ}$ mês $(p<0.05)$. Na - Figura 2 pode-se observar a distribuição dos tempos de avaliação do WOMAC no grupo estudo.

Em relação ao grupo do $\mathrm{AH}$, houve uma diferença estatística no escore de WOMAC entre o resultado inicial e com 1 mês $(p<0.05)$ e o resultado inicial e com 3 meses $(p<0.05)$. Não

Tabela 2 Comparação entre os grupos através do escore do Western Ontario and McMaster Universities Osteoarthritis Index *

\begin{tabular}{|l|l|l|l|l|l|}
\hline \multicolumn{2}{|l|}{} & WOMAC Inicial & WOMAC 1 mês & WOMAC 3 Meses & WOMAC 6 meses \\
\hline PRP & Média & 42.5 & 29.0 & 23.7 & 41.1 \\
\hline & Desvio padrão & 17.9 & 16.0 & 22.0 & 24.8 \\
\hline $\mathrm{AH}$ & Média & 41.1 & 24.0 & 26.0 & 35.7 \\
\hline & Desvio padrão & 15.5 & 14.6 & 22.0 & 35.7 \\
\hline & Valor de $p$ & 0.82 & 0.39 & 0.78 & 0.73 \\
\hline
\end{tabular}

Abreviaturas: AH, ácido hialurônico; PRP, plasma rico em plaquetas; WOMAC, Western Ontario and McMaster Universities Osteoarthritis Index. Fonte: Prevent Senior São Paulo. 


\section{WOMAC - Grupo PRP}

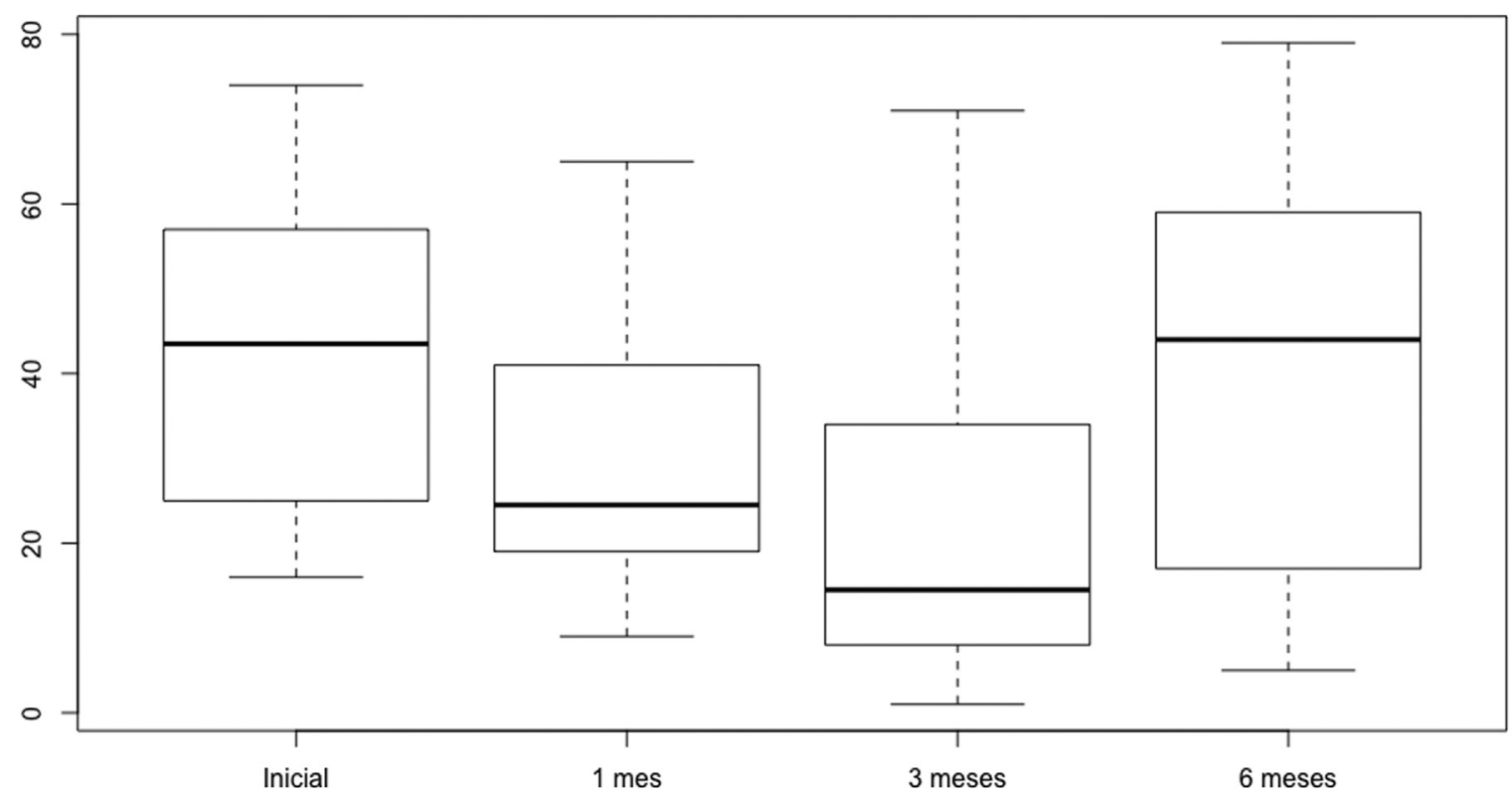

Fig. 2 Distribuição do escore do Western Ontario and McMaster Universities Osteoarthritis Index no grupo plasma rico em plaquetas.

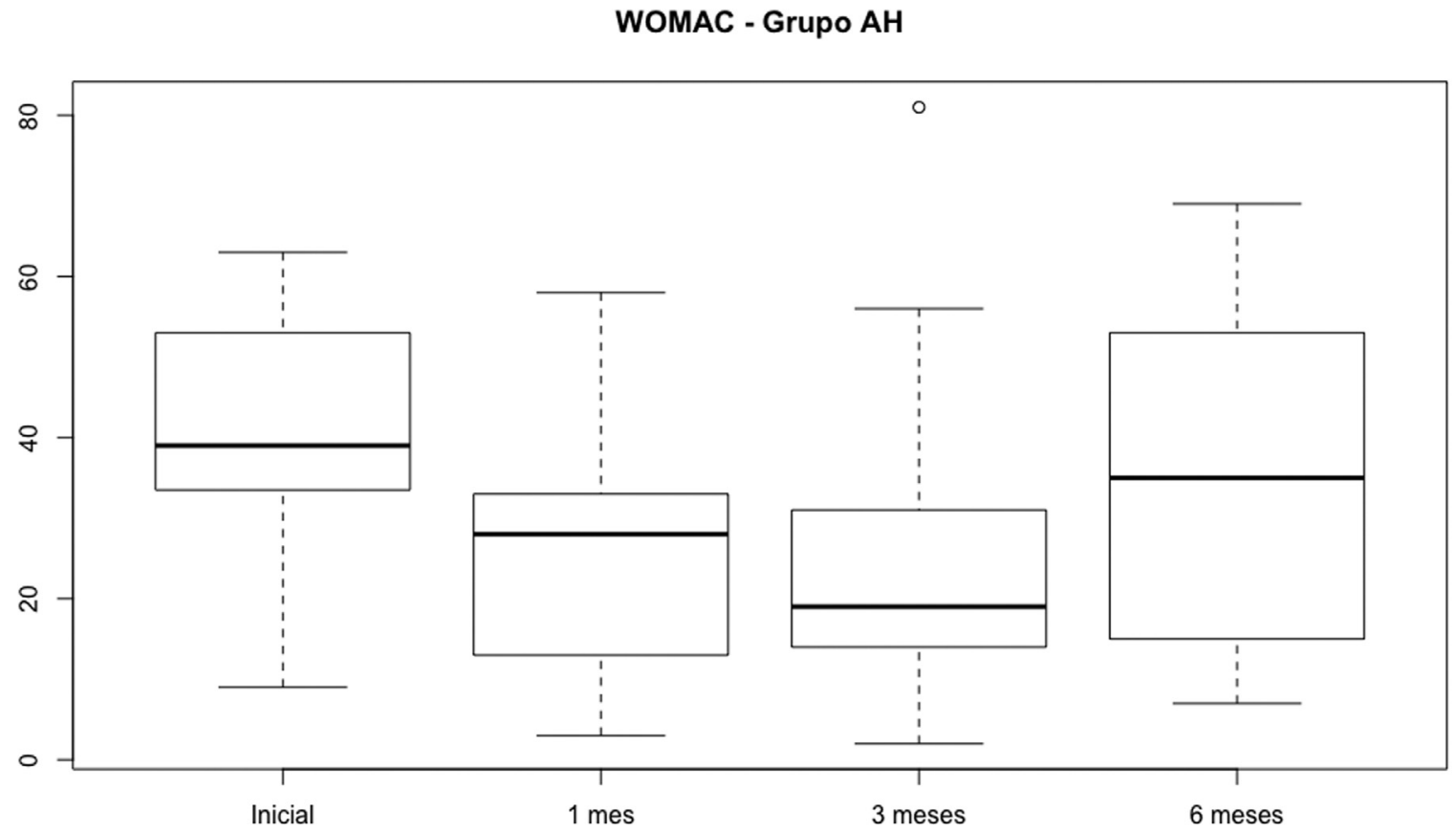

Fig. 3 Distribuição do escore do Western Ontario and McMaster Universities Osteoarthritis Index no grupo ácido hialurônico.

houve diferença estatística em relação à dor do $2^{\circ}$ e $4^{\circ}$ meses $(p=0.49)$ pela EVA. A - Figura 3 revela essa distribuição

Não houve nenhum caso de infecção ou alguma reação alérgica durante os 6 meses de acompanhamento dos pacientes. Os casos de dor foram tratados com medicações analgésicas, crioterapia e reabilitação.

\section{Discussão}

O principal achado do estudo foi a ausência de diferença entre os resultados funcionais e avaliação de dor com acompanha- mento a médio prazo ( 6 meses) dos pacientes submetidos à infiltração intraarticular de AH e de PRP. No entanto, ambos os métodos de tratamento foram efetivos na melhora da dor e função dos pacientes ao longo do período estudado e se mostraram seguros na presente amostra.

A avaliação funcional dos pacientes foi realizada utilizando o questionário WOMAC e não foram observadas diferenças entre os dois grupos estudados ao longo dos 6 meses de acompanhamento. A literatura ainda é controversa em relação a este desfecho. Uma revisão sistemática publicada recentemente, utilizando o WOMAC total na avaliação funcional, 
concluiu que o PRP é superior ao AH nos estudos com acompanhamento de médio prazo (3-6 meses). Porém, o mesmo estudo não encontrou diferenças entre os grupos quando analisaram os escores fracionados do WOMAC para rigidez e função física. ${ }^{16}$

Outra metanálise demonstrou superioridade do PRP em relação ao AH na melhora da dor avaliada pelo WOMAC. No entanto, o estudo concluiu não existir uma superioridade óbvia entre os grupos no tratamento da osteoartrose do joelho. ${ }^{17}$

Alguns ensaios clínicos randomizados comparando estes dois métodos no tratamento da $\mathrm{OA}$ também não encontraram diferenças nos escores funcionais após 6 meses de acompanhamento. ${ }^{18,19}$

A avaliação do quadro álgico dos pacientes no presente estudo não encontrou diferenças entre os grupos avaliados (PRP x AH). Da mesma forma, os resultados são bem divergentes na literatura. Zhang et al. ${ }^{17}$ também não encontraram diferenças na EVA da dor entre os 2 tratamentos no período de 3 e 6 meses após infiltrações. Entretanto, Cole et al. ${ }^{19}$ demonstraram melhora significativa da dor medida pela EVA no grupo de pacientes tratados com PRP após 6 e 12 meses de infiltração.

A maioria das revisões sistemáticas sobre o assunto relata grande dificuldade de comparação entre os diversos estudos publicados devido a grandes variações na metodologia do preparo e na composição do PRP, número de aplicações realizadas, estudos com amostras pequenas e seguimentos curtos e com diferentes critérios de inclusão e avaliação. ${ }^{20}$

Nosso estudo utilizou um kit padronizado para preparo do PRP, amostras homogêneas e uma infiltração semanal por 3 semanas. Estudos prévios haviam mostrado vantagens das múltiplas aplicações de PRP quando comparadas com infiltração isolada. Inclusive mostrando efeitos mais prolongados da ação do PRP quando eram realizadas mais de uma aplicação. $^{21,22}$

O presente estudou demonstrou que ambos os tratamentos realizados (PRP e AH) foram efetivos no tratamento da dor e na melhora da função dos pacientes. No entanto, o efeito das medicações vai deteriorando com o tempo e praticamente esgota-se após 5 a 6 meses após o tratamento. Di Martino et al., ${ }^{23}$ num ensaio clínico randomizado, encontraram resultados semelhantes. No estudo, os pacientes relatavam melhora dos sintomas até 9 meses após a aplicação de $A H$ e até 12 meses após a infiltração intraarticular com PRP, mostrando, desta maneira, perda progressiva dos efeitos proporcionados pelos tratamentos.

Filardo et al. ${ }^{18}$ também encontraram resultados semelhantes num ensaio clínico randomizado com um ano de follow-up. Neste estudo, os autores mostraram melhora do quadro álgico e função nos grupos de pacientes tratados com PRP e AH, mas os resultados mantiveram-se praticamente estáveis após 2 meses do tratamento.

Em relação aos efeitos adversos de ambos os tratamentos, tanto o PRP quanto o AH mostraram-se seguros em nossa amostra. Nenhum dos medicamentos apresentou efeitos colaterais severos e duradouros. Han et al., em uma metanálise, não encontraram diferenças entre os grupos de tratamento em relação aos efeitos adversos. ${ }^{24}$ Outros estudos também concluíram que os dois métodos de tratamento são seguros e com poucos efeitos colaterais observados durante o período de acompanhamento. ${ }^{25,26}$

0 presente estudo apresenta algumas limitações. Primeiramente, apesar de se tratar de um estudo apresentado com resultados preliminares, a amostra é pequena. Depois, o período de acompanhamento foi relativamente curto (6 meses), e alguns estudos apresentam resultados mais prolongados do PRP quando comparado ao AH. A ausência de um grupocontrole (placebo ou corticoide) e a falta de cegamento dos grupos são outras limitações do presente estudo.

\section{Conclusão}

A infiltração intraarticular com AH ou PRP nos joelhos dos pacientes com gonartrose primária apresentou melhora temporária dos sintomas de dor e função, sendo ambas as técnicas seguras. Não houve diferença entre as duas intervenções.

\section{Suporte Financeiro}

Não houve suporte financeiro de fontes públicas, comerciais, ou sem fins lucrativos.

Conflito de interesses

Os autores declaram não haver conflito de interesses.

\section{Referências}

1 Keyes GW, Carr AJ, Miller RK, Goodfellow JW. The radiographic classification of medial gonarthrosis. Correlation with operation methods in 200 knees. Acta Orthop Scand 1992;63(05): 497-501

2 Kellgren JH, Lawrence JS. Radiological assessment of osteo-arthrosis. Ann Rheum Dis 1957;16(04):494-502

3 Ahlbäck S. Osteoarthrosis of the knee. A radiographic investigation. Acta Radiol Diagn (Stockh) 1968(Suppl 277):277-272, 7-72

4 Lana JF, Weglein A, Sampson SE, et al. Randomized controlled trial comparing hyaluronic acid, platelet-rich plasma and the combination of both in the treatment of mild and moderate osteoarthritis of the knee. J Stem Cells Regen Med 2016;12(02):69-78

5 Schnabel LV, Mohammed HO, Miller BJ, et al. Platelet rich plasma (PRP) enhances anabolic gene expression patterns in flexor digitorum superficialis tendons. J Orthop Res 2007;25(02): 230-240

6 Fortier LA, Hackett CH, Cole BJ. The effects of platelet-rich plasma on cartilage: basic science and clinical application. Oper Tech Sports Med 2011;19:154-159

7 Steinert AF, Rackwitz L, Gilbert F, Nöth U, Tuan RS. Concise review: the clinical application of mesenchymal stem cells for musculoskeletal regeneration: current status and perspectives. Stem Cells Transl Med 2012;1(03):237-247

8 Research Randomizer. (Version 4.02013) [computer program]. http://www.randomizer.org/

9 R: A language and environment for statistical computing [computer program]. Vienna, Austria: R Foundation for Statistical; 2014

10 Shapiro SS, Wilk MB. An analysis of variance test for normality. Biometrika 1965;52(3-4):591-611

11 Fisher RA. The Correlation Between Relatives on the Supposition of Mendelian Inheritance. Philos Trans Royal Soc Edinburgh. 1918;52:399-433 
12 Gosset SWS. The probable error of a mean. Biometrika 1908;6 (01):1-25

13 Neuhäuser M. Wilcoxon-Mann-Whitney Test. In: Lovric M, editor. International Encyclopedia of Statistical Science. Berlin, Heidelberg: Springer Berlin Heidelberg; 2011:1656-1658

14 Pearson KX. On the criterion that a given system of deviations from the probable in the case of a correlated system of variables is such that it can be reasonably supposed to have arisen from random sampling. Lond Edinb Philos Mag J Sci 1900;50(302): 157-175

15 Fisher RA. On the Interpretation of $X^{2}$ from Contingency Tables, and the Calculation of P. J R Stat Soc 1922;85(01):87-94

16 Chen Z, Wang C, You D, Zhao S, Zhu Z, Xu M. Platelet-rich plasma versus hyaluronic acid in the treatment of knee osteoarthritis: $A$ meta-analysis. Medicine (Baltimore) 2020;99(11):e19388

17 Zhang HF, Wang CG, Li H, Huang YT, Li ZJ. Intra-articular platelet-rich plasma versus hyaluronic acid in the treatment of knee osteoarthritis: a meta-analysis. Drug Des Devel Ther 2018;12:445-453

18 Filardo G, Di Matteo B, Di Martino A, et al. Platelet-Rich Plasma Intra-articular Knee Injections Show No Superiority Versus Viscosupplementation: A Randomized Controlled Trial. Am J Sports Med 2015;43(07):1575-1582

19 Cole BJ, Karas V, Hussey K, Pilz K, Fortier LA. Hyaluronic Acid Versus Platelet-Rich Plasma: A Prospective, Double-Blind Randomized Controlled Trial Comparing Clinical Outcomes and Effects on Intra-articular Biology for the Treatment of Knee Osteoarthritis. Am J Sports Med 2017;45(02):339-346
20 Cook CS, Smith PA. Clinical Update: Why PRP Should Be Your First Choice for Injection Therapy in Treating Osteoarthritis of the Knee. Curr Rev Musculoskelet Med 2018;11(04):583-592

21 Chouhan DK, Dhillon MS, Patel S, Bansal T, Bhatia A, Kanwat H. Multiple Platelet-Rich Plasma Injections Versus Single PlateletRich Plasma Injection in Early Osteoarthritis of the Knee: An Experimental Study in a Guinea Pig Model of Early Knee Osteoarthritis. Am J Sports Med 2019;47(10):2300-2307

22 Görmeli G, Görmeli CA, Ataoglu B, Colak C, Aslantürk O, Ertem K. Multiple PRP injections are more effective than single injections and hyaluronic acid in knees with early osteoarthritis: a randomized, double-blind, placebo-controlled trial. Knee Surg Sports Traumatol Arthrosc 2017;25(03):958-965

23 Di Martino A, Di Matteo B, Papio T, et al. Platelet-Rich Plasma Versus Hyaluronic Acid Injections for the Treatment of Knee Osteoarthritis: Results at 5 Years of a Double-Blind, Randomized Controlled Trial. Am J Sports Med 2019;47(02):347-354

24 Han Y, Huang H, Pan J, et al. Meta-analysis Comparing PlateletRich Plasma vs Hyaluronic Acid Injection in Patients with Knee Osteoarthritis. Pain Med 2019;20(07):1418-1429

25 Chen P, Huang L, Ma Y, et al. Intra-articular platelet-rich plasma injection for knee osteoarthritis: a summary of meta-analyses. J Orthop Surg Res 2019;14(01):385

26 Di Y, Han C, Zhao L, Ren Y. Is local platelet-rich plasma injection clinically superior to hyaluronic acid for treatment of knee osteoarthritis? A systematic review of randomized controlled trials. Arthritis Res Ther 2018;20(01):128 